\title{
SENSITIVE DETERMINATION OF RELATED SUBSTANCES IN PIOGLITAZONE HYDROCHLORIDE BY HPLC
}

\author{
N. BALAJI ${ }^{*}$, SAYEEDA SULTANA ${ }^{2}$ \\ ${ }^{1,2}$ Department of Chemistry, St. Peter's University, Avadi, Chennai 600054, Tamil Nadu, India \\ Email: priyabalan8380@gmail.com
}

Received: 28 Dec 2016, Revised and Accepted: 02 Mar 2017

\section{ABSTRACT}

Objective: An efficient, high performance liquid chromatographic method has been developed and validated for the quantification of related substances in pioglitazone hydrochloride drug substance.

Methods: This method includes the determination of three related substances in pioglitazone hydrochloride. The mobile phase $\mathrm{A}$ is $0.1 \% \mathrm{w} / \mathrm{v}$ triethylamine in water with $\mathrm{pH} 2.5$ adjusted by dilute phosphoric acid. The mobile phase B is premixed and degassed mixtures of acetonitrile and methanol. The flow rate was $1 \mathrm{ml} / \mathrm{min}$. The elution used was gradient mode. The HPLC column used for the analysis was symmetry C18 with a length of $250 \mathrm{~mm}$, the internal diameter of $4.6 \mathrm{~mm}$ and particle size of 5.0 microns.

Results: The developed method was found to be linear with the range of $0.006-250 \%$ with a coefficient of correlation 0.99 . The precision study revealed that the percentage relative standard deviation was within the acceptable limit. The limit of detection and limit of quantitation of the impurities was less than $0.002 \%$ and $0.006 \%$ with respect to pioglitazone hydrochloride test concentration of $2000 \mu \mathrm{g} / \mathrm{ml}$ respectively. This method has been validated as per ICH guidelines Q2 (R1).

Conclusion: A reliable, economical HPLC method was magnificently established for quantitative analysis of related substances of pioglitazone hydrochloride drug substance.

Keywords: Pioglitazone hydrochloride, Related substances, HPLC, Validation

(C) 2016 The Authors. Published by Innovare Academic Sciences Pvt Ltd. This is an open access article under the CC BY license (http://creativecommons.org/licenses/by/4.0/) DOI: http://dx.doi.org/10.22159/ijap.2017v9i2.16828

\section{INTRODUCTION}

Pioglitazone is a prescription drug of the thiazolidinedione class with hypoglycemic action to treat diabetes. Pioglitazone is used to lower blood glucose levels in the treatment of diabetes mellitus type 2 either alone or in combination with a sulfonylurea, metformin, or insulin. Pioglitazone has also been used to treat non-alcoholic steatohepatitis [1-2].

The related substances of pioglitazone hydrochloride have been developed and validated as per the selection of the synthetic route. Nowadays, the regulators were very much interested about the strategy of the control of impurities present in drug substances. They insist to have a regulation on impurities in each step of the synthetic drug process. Many studies were explained about the determination of pioglitazone was performed by HPLC [3-25, 27-31]. So as to determine the related substances of pioglitazone hydrochloride, the research work has been initiated.

Several methods have been developed and validated only for the content of pioglitazone hydrochloride in drug substances or drug products and not for their related substances [3-25, 28-31]. This has been triggered us to perform the development activity for the determination of related substances in pioglitazone hydrochloride by HPLC and their structures were shown in fig. 1. As per the literature survey of the pioglitazone hydrochloride, no one has reported the most sensitive method for the determination of three impurities in pioglitazone hydrochloride drug substance by HPLC. These three impurities were determined at very low-level detection and quantitation in pioglitazone hydrochloride drug substance and this comprises the novelty of the article.

\section{MATERIALS AND METHODS}

Pioglitazone hydrochloride, PGR-II, PIO-II, N-oxide were gifted by Techno chemicals. The structure of related substances and pioglitazone hydrochloride has shown in fig. 1. Triethylamine, methanol, phosphoric acid and acetonitrile were bought from Fisher scientific. HPLC grade water was used, equipped with the Elga water purification system, Metrohm. Transferred $1 \mathrm{ml}$ of triethylamine in $1000 \mathrm{ml}$ water, adjusted the $\mathrm{pH}$ of the solution to 2.5 using dilute orthophosphoric acid, filtered and degassed [27-31]. This solution was named as mobile phase-A.
Mixed $800 \mathrm{ml}$ of acetonitrile and $200 \mathrm{ml}$ of methanol, degassed and used as mobile phase-B. Water bath equipped with a controller (Amkette analytics, ANM alliance) was used for forced degradation studies. Photolytic studies were carried out in a photostability chamber (Thermolab photostability chamber, India). Thermal degradation works were accomplished in a hot air oven (Amkette analytics, ANM alliance).
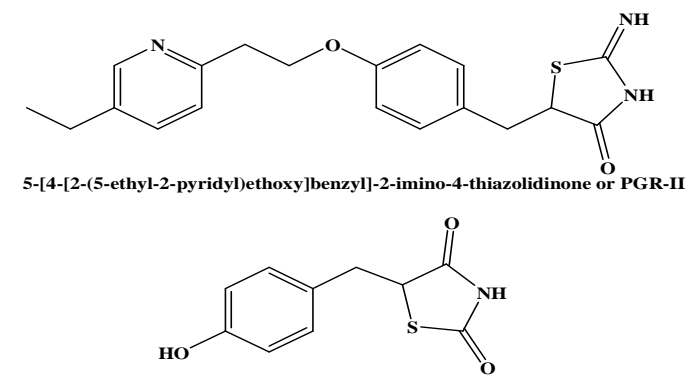

5-(4-Hydroxy-benzyl)-thiazolidine-2,4-dione or PIO-II

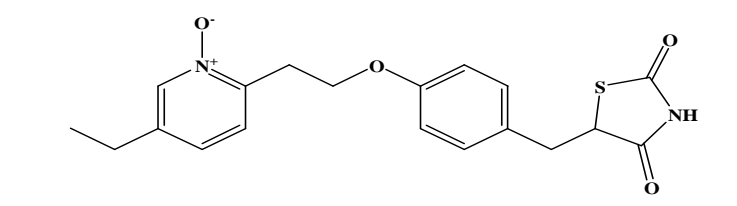

5-\{4-[2-(5-Ethyl-1-oxy-pyridin-2-yl)-ethoxy]-benzyl\}-thiazolidine-2,4-dione or N-Oxide

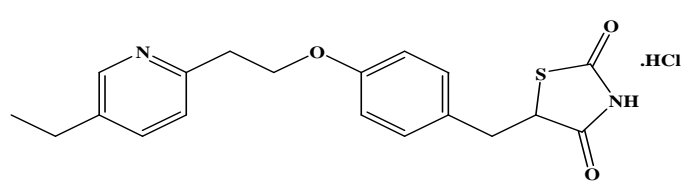
5-\{4-[2-(5-Ethyl-pyridin-2-yl)-ethoxy]-benzyl\}-thiazolidine-2,4-dione, hydrochloride or Pioglitazone

Fig. 1: The structures of related substances and pioglitazone hydrochloride 


\section{Preparation of solutions}

The sample diluent was prepared by diluting $8.5 \mathrm{ml}$ of concentrated hydrochloric acid in $1000 \mathrm{ml}$ of water. Filtered and degassed for usage of analysis. The system suitability solution was prepared by exactly weighed and transferred about $10 \mathrm{mg}$ each of PIO-II, PGR-II, $\mathrm{N}$-oxide and pioglitazone standard in $100 \mathrm{ml}$ volumetric flask. Dissolved and make upto the volume $100 \mathrm{ml}$ with sample diluent.
Further, $2 \mathrm{ml}$ of this solution was diluted into a $100 \mathrm{ml}$ volumetric flask and made up to the mark with sample diluent. So, the standard solution concentration was $100 \mu \mathrm{g} / \mathrm{ml}$ with respect to the test concentration of $2000 \mu \mathrm{g} / \mathrm{ml}$ pioglitazone hydrochloride. The sample solution was prepared by accurately weighed and transferred about $100 \mathrm{mg}$ of sample into $50 \mathrm{ml}$ volumetric flask. Dissolved and make upto the volume $50 \mathrm{ml}$ with sample diluent. The chromatograph of system suitability solution has been shown in fig. 2 .

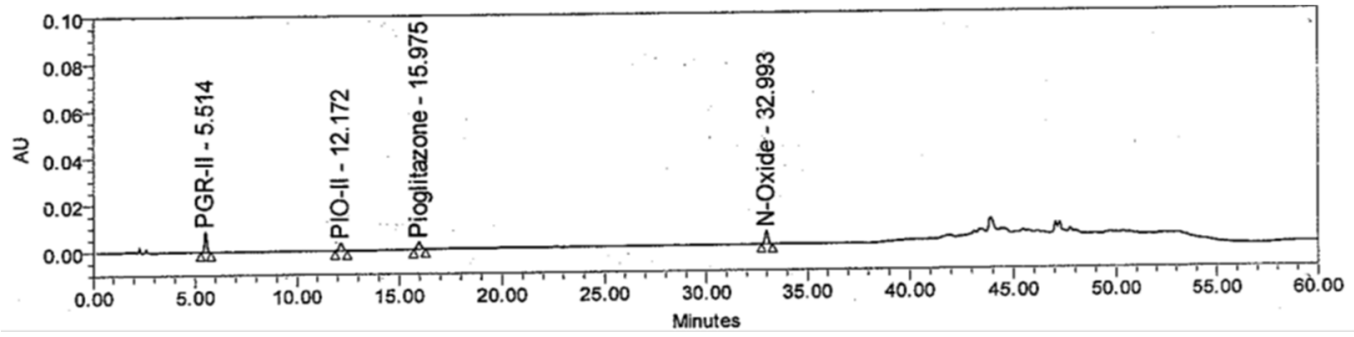

Fig. 2: Chromatograms of system suitability solution

\section{Concluded method for validation purpose}

The HPLC column was used symmetry C18, $250 \mathrm{~mm}$ x $4.6 \mathrm{~mm} \times 5.0$ $\mu \mathrm{m}$. Transferred $1 \mathrm{ml}$ of triethylamine in $1000 \mathrm{ml}$ water, adjusted the $\mathrm{pH}$ of the solution to 2.5 using dilute orthophosphoric acid, filtered and degassed. This solution was named as mobile phase-A. Mixed $800 \mathrm{ml}$ of acetonitrile and $200 \mathrm{ml}$ of methanol, degassed and used as mobile phase-B. The gradient program was mentioned as min/\%B composition; 0.00/20.0, 10.00/20.0, 35.00/40.0, 40.00/ $80.0,50 / 80.0,51.00 / 20.0$ and $60.00 / 20.0$. The flow rate was 1 $\mathrm{ml} / \mathrm{min}$. The wavelength of detection was $225 \mathrm{~nm}$ and the injection volume was $10 \mu \mathrm{l}$. The column compartment temperature was maintained at $45^{\circ} \mathrm{C}$.

\section{RESULTS}

\section{Analytical method development}

Several methods have been developed by HPLC for the determination of pioglitazone in the bulk and formulated products [3-25, 28-31]. These previously published research articles were failed to explain about the determination of related substances in pioglitazone. This mainly leads us to do further development of the method with three impurities in pioglitazone by HPLC.

The same column [3] has been used in the initial development. So, as to develop the sensitive method, the mobile phase, $\mathrm{pH}$ and gradient composition have been modified accordingly. The $\mathrm{pH}$ of the mobile phase was maintained in the acidic region, which was achieved by the addition of $1 \mathrm{ml}$ of triethylamine in $1000 \mathrm{ml}$ of water, adjusted the solution $\mathrm{pH}$ to 2.5 using dilute phosphoric acid. Generally, the mass compatible mobile phase needs to be selected, because if any impurities were detected at the level of above or below LOQ in the sample which needs to be confirmed using HPLC-MS study. But the mobile phase chosen for the study was not compatible with mass spectrometry (MS). So, this could be the limitation of the present research work. These three impurities have been eluted and separated well within the time of $40 \mathrm{~min}$., but to ensure the consistency and specificity of the other impurities which was mentioned in the research article [27-31]; the gradient program has been slightly modified and extended up to $60 \mathrm{~min}$. From this trial, the method was specific for all the impurities which have mentioned in the research article [27-31]. The results and comparisons of previously published articles were given in table 1 .

Table 1: Reported analytical HPLC methods for determination of pioglitazone (PIO) either alone or in combination with other drugs like metformin (MET), glimepiride (GLM), rosiglitazone (ROS) and gliclazide (GLC) in pharmaceutical dosage forms.

\begin{tabular}{|c|c|c|c|c|c|c|}
\hline Reference & Study Aim & Mobile phase & Column & $\begin{array}{l}\text { Wavelength } \\
\text { (nm) }\end{array}$ & $\begin{array}{l}\text { Flow rate } \\
\text { (ml/min) }\end{array}$ & LOD $(\mu \mathrm{g} / \mathrm{ml})$ \\
\hline 3 & $\begin{array}{l}\text { In bulk and pharmaceutical } \\
\text { formulations by HPLC and } \\
\text { MEKC method }\end{array}$ & $\begin{array}{l}0.01 \mathrm{M} \mathrm{KH}_{2} \mathrm{PO}_{4} \text { buffer }(\mathrm{pH} \\
6.0): \mathrm{ACN}(50: 50, \mathrm{v} / \mathrm{v})\end{array}$ & $\begin{array}{l}\text { Symmetry C18 (250 } \\
\mathrm{mm} \times 4.6 \mathrm{mmx} 5 \mu \mathrm{m})\end{array}$ & 225 & 1 & - \\
\hline 4 & SIAM by RP-HPLC & $\begin{array}{l}\text { Phosphate buffer (pH } \\
4.0), A C N \text { and methanol } \\
(55: 30: 15, v / v)\end{array}$ & $\begin{array}{l}\text { Prontosil C8 }(250 \mathrm{~mm} \\
\mathrm{x} 4.6 \mathrm{~mm} \times 5 \mu \mathrm{m})\end{array}$ & 254 & 1.5 & - \\
\hline 5 & $\begin{array}{l}\text { Study of stressed } \\
\text { degradation behaviour in } \\
\text { bulk and pharmaceutical } \\
\text { formulation }\end{array}$ & $\begin{array}{l}0.01 \mathrm{M} \mathrm{KH}_{2} \mathrm{PO}_{4} \text { buffer }(\mathrm{pH} \\
3.5) \text { and methanol }(55: 45 \text {, } \\
\mathrm{v} / \mathrm{v})\end{array}$ & $\begin{array}{l}\text { Phenomenex Luna } \\
\text { C18 }(250 \mathrm{~mm} \times 4.6 \\
\mathrm{mm} \times 5 \mu \mathrm{m})\end{array}$ & 241 & 1.5 & 1.69 \\
\hline 6 & Assay of tablets & $\begin{array}{l}\text { Ammonium formate } \\
\text { buffer }(\mathrm{pH} \mathrm{3}) \text { : ACN } \\
(75: 25, \mathrm{v} / \mathrm{v})\end{array}$ & $\begin{array}{l}\text { Nova-Pak C18 }(150 \\
\mathrm{mm} \times 3.9 \mathrm{~mm} \times 5 \mu \mathrm{m})\end{array}$ & 225 & 1 & - \\
\hline 7 & $\begin{array}{l}\text { Purity test and assay of } \\
\text { tablets }\end{array}$ & $\begin{array}{l}\text { Ammonium formate } \\
\text { buffer }(\mathrm{pH} 4.1) \text { : ACN } \\
(45: 55, \mathrm{v} / \mathrm{v})\end{array}$ & $\begin{array}{l}\text { Symmetry C18 }(250 \\
\mathrm{mm} \times 4.6 \mathrm{~mm} \times 5 \mu \mathrm{m})\end{array}$ & 266 & 1 & 0.042 \\
\hline 8 & SIAM & $\begin{array}{l}\text { ACN: }(0.15, \mathrm{v} / \mathrm{v}) \\
\text { triethylamine }(\mathrm{pH} 4.6) \\
(40: 60, \mathrm{v} / \mathrm{v})\end{array}$ & $\begin{array}{l}\text { Hypersil C-8 }(250 \\
\mathrm{mm} \times 4.6 \mathrm{~mm} \text { x } 5 \mu \mathrm{m})\end{array}$ & 220 & 1.5 & 0.6 \\
\hline 9 & $\begin{array}{l}\text { Simultaneous determination } \\
\text { with GLM }\end{array}$ & $\begin{array}{l}0.01 \mathrm{M} \text { triammonium } \\
\text { citrate (pH 6.95):ACN: } \\
\text { MeOH }(45: 35: 20, \mathrm{v} / \mathrm{v} / \mathrm{v})\end{array}$ & $\begin{array}{l}\text { Cosmosil C18 }(150 \\
\mathrm{mm} \times 4.6 \mathrm{~mm} \times 5 \mu \mathrm{m})\end{array}$ & 228 & 1 & - \\
\hline 10 & Simultaneous with MET & $\begin{array}{l}\text { ACN: } \mathrm{KH}_{2} \mathrm{PO}_{4} \text { buffer }(\mathrm{pH} \\
\text { 3) }(50: 50, v / v)\end{array}$ & $\begin{array}{l}\text { Hypersil BDS C18 } \\
(250 \mathrm{~mm} \times 4.6 \mathrm{~mm} \times \\
5 \mu \mathrm{m})\end{array}$ & 238 & 1 & - \\
\hline
\end{tabular}




\begin{tabular}{|c|c|c|c|c|c|c|}
\hline 11 & $\begin{array}{l}\text { Simultaneous determination } \\
\text { with GLM }\end{array}$ & $\begin{array}{l}\text { ACN: } 0.02 \text { M Ammonium } \\
\text { acetate buffer }(\mathrm{pH} 4.5) \\
(60: 40, \mathrm{v} / \mathrm{v})\end{array}$ & $\begin{array}{l}\text { Inertsil ODS }(250 \mathrm{~mm} \\
\times 4.6 \mathrm{~mm} \times 5 \mu \mathrm{m})\end{array}$ & 230 & 1 & 0.2 \\
\hline 12 & $\begin{array}{l}\text { Simultaneous determination } \\
\text { with MET and GLM in tablet } \\
\text { formulation }\end{array}$ & $\begin{array}{l}\mathrm{MeOH}: \mathrm{KH}_{2} \mathrm{PO}_{4} \text { buffer } \\
(\mathrm{pH} \mathrm{4.3)}(75: 25, \mathrm{v} / \mathrm{v})\end{array}$ & $\begin{array}{l}\text { Inertsil ODS-3 C18 } \\
(250 \mathrm{~mm} \times 4.6 \mathrm{~mm} \times \\
5 \mu \mathrm{m})\end{array}$ & 258 & 1 & - \\
\hline 13 & $\begin{array}{l}\text { Simultaneous determination } \\
\text { with saxagliptin in tablets }\end{array}$ & $\begin{array}{l}\text { ACN: } 0.02 \mathrm{M} \mathrm{KH}_{2} \mathrm{PO}_{4} \text { buffer } \\
(\mathrm{pH} 7.0)(60: 40, \mathrm{v} / \mathrm{v})\end{array}$ & $\begin{array}{l}\text { Inertsil C18 }(150 \mathrm{~mm} \\
\times 4.6 \mathrm{~mm} \times 5 \mu \mathrm{m})\end{array}$ & 260 & 0.8 & 0.010 \\
\hline 14 & $\begin{array}{l}\text { Simultaneous determination } \\
\text { with GLM }\end{array}$ & $\begin{array}{l}\text { ACN: } 0.01 \mathrm{M} \mathrm{KH}_{2} \mathrm{PO}_{4} \text { buffer } \\
(\mathrm{pH} 6.2)(50: 50, \mathrm{v} / \mathrm{v})\end{array}$ & $\begin{array}{l}\text { Eurosphere- } 100 \mathrm{C} 18 \\
(250 \mathrm{~mm} \times 4.6 \mathrm{~mm} \times \\
5 \mu \mathrm{m})\end{array}$ & 225 & 1.4 & 0.00049 \\
\hline 15 & $\begin{array}{l}\text { Simultaneous determination } \\
\text { with GLM and ROS }\end{array}$ & $\begin{array}{l}\text { Dil. } \mathrm{H}_{3} \mathrm{PO}_{4}(\mathrm{pH} 3.0): \mathrm{ACN} \\
(80: 20, \mathrm{v} / \mathrm{v})\end{array}$ & $\begin{array}{l}\text { Nucleodur C-18 }(250 \\
\mathrm{mm} \times 4.6 \mathrm{~mm} \times 5 \mu \mathrm{m})\end{array}$ & 215 & 0.8 & 0.19 \\
\hline 16 & $\begin{array}{l}\text { Estimation along with MET } \\
\text { in tablets }\end{array}$ & $\begin{array}{l}\text { ACN: water: acetic acid } \\
(75: 25: 0.3, v / v / v), \text { pH } 5.5\end{array}$ & $\begin{array}{l}\text { Hypersil ODS C18 } \\
(250 \mathrm{~mm} \times 4.6 \mathrm{~mm} \times \\
5 \mu \mathrm{m})\end{array}$ & 230 & 0.5 & 0.009 \\
\hline 17 & $\begin{array}{l}\text { Simultaneous quantification } \\
\text { with GLM and MET }\end{array}$ & $\begin{array}{l}\text { ACN: } 0.01 \mathrm{M} \mathrm{KH}_{2} \mathrm{PO}_{4} \\
\text { buffer-pH 5.0:THF } \\
(50: 40: 10, \mathrm{v} / \mathrm{v} / \mathrm{v})\end{array}$ & $\begin{array}{l}\text { Inertsil ODS 3V ( } 250 \\
\mathrm{~mm} \times 4.6 \mathrm{~mm} \times 5 \mu \mathrm{m})\end{array}$ & 228 & 1.7 & - \\
\hline 18 & $\begin{array}{l}\text { Simultaneous estimation } \\
\text { along with GLM }\end{array}$ & $\begin{array}{l}\text { ACN: } \mathrm{KH}_{2} \mathrm{PO}_{4} \text { buffer } \\
(60: 40, \mathrm{v} / \mathrm{v})\end{array}$ & $\begin{array}{l}\text { Inertsil ODS }(150 \mathrm{~mm} \\
\times 4.6 \mathrm{~mm} \times 5 \mu \mathrm{m}))\end{array}$ & 225 & 1.5 & 0.12 \\
\hline 19 & SIAM along with GLM & $\begin{array}{l}\text { Solution A: ACN } \\
\text { Solution B: } 0.02 \mathrm{M} \\
\mathrm{KH}_{2} \mathrm{PO}_{4} \text { buffer (pH: } 3.2 \text { ) }\end{array}$ & $\begin{array}{l}\text { Zorbax cyano }(250 \\
\mathrm{mm} \times 4.6 \mathrm{~mm} \times 5 \mu \mathrm{m})\end{array}$ & 230 & 0.8 & - \\
\hline 20 & $\begin{array}{l}\text { Simultaneous determination } \\
\text { with MET and GLC in } \\
\text { multicomponent formulation }\end{array}$ & $\begin{array}{l}\text { MeOH: } 0.02 \mathrm{M} \mathrm{KH}_{2} \mathrm{PO}_{4} \\
\text { buffer }(85: 15, \mathrm{v} / \mathrm{v})\end{array}$ & $\begin{array}{l}\text { HiQSilC18 HS ( } 250 \\
\mathrm{~mm} \times 4.6 \mathrm{~mm} \times 5 \mu \mathrm{m})\end{array}$ & 227 & 1.2 & 0.1 \\
\hline 21 & $\begin{array}{l}\text { Simultaneous estimation } \\
\text { with GLM }\end{array}$ & $\begin{array}{l}\text { Methanol: water }(72: 28, \\
\text { v/v) }\end{array}$ & $\begin{array}{l}\text { AgilentTC-C18 (250 } \\
\left.\mathrm{mm} \_4.6 \mathrm{~mm}, 5 \mu \mathrm{m}\right)\end{array}$ & 230 & 1 & 0.760 \\
\hline 22 & $\begin{array}{l}\text { Simultaneous estimation } \\
\text { with telmisartan }\end{array}$ & $\begin{array}{l}\text { ACN: ammonium } \\
\text { dihydrogen phosphate }(\mathrm{pH} \\
4.5 ; 0.02 \mathrm{M})(65: 35, \mathrm{v} / \mathrm{v})\end{array}$ & $\begin{array}{l}\text { Phenomenex C8 }(250 \\
\text { mm_4.6 mm,5 } \mu \mathrm{m})\end{array}$ & 210 & 1 & 0.82 \\
\hline 23 & $\begin{array}{l}\text { Determination of along with } \\
\text { MET and GLM }\end{array}$ & $\begin{array}{l}\text { ACN: phosphate buffer } \\
(\mathrm{pH} 3)(65: 35, \mathrm{v} / \mathrm{v})\end{array}$ & $\begin{array}{l}\text { PhenomenexRP-18 } \\
\left(150 \mathrm{~mm} \_4.6 \mathrm{~mm}, 5\right. \\
\mu \mathrm{m})\end{array}$ & 245 & 0.5 & 0.061 \\
\hline 24 & $\begin{array}{l}\text { Micellar liquid } \\
\text { chromatographic analytical } \\
\text { method for determination of } \\
\text { atorvastatin calcium }\end{array}$ & $\begin{array}{l}\text { Tween-20: n-butanol: } \\
\text { phosphate buffer, }(\mathrm{pH} \\
\text { 4.2) }(50: 25: 25, \mathrm{v} / \mathrm{v} / \mathrm{v})\end{array}$ & $\begin{array}{l}\text { Luna C18 }(250 \\
\left.\mathrm{mm}_{-} 4.6 \mathrm{~mm}, 5 \mu \mathrm{m}\right)\end{array}$ & 322 & 1.5 & - \\
\hline 25 & HPLC & $\begin{array}{l}0.01 \mathrm{M} \text { buffer, } \mathrm{pH}- \\
6.0: \text { methanol }(40: 60, \mathrm{v} / \mathrm{v})\end{array}$ & $\begin{array}{l}\text { Symmetry-extend- } \\
\text { C18 (150 mm_4.6 } \\
\mathrm{mm}, 5 \mu \mathrm{m})\end{array}$ & 240 & 1.2 & - \\
\hline 27 & $\begin{array}{l}\text { SIAM for determination of } \\
\text { impurities in PIO }\end{array}$ & $\begin{array}{l}\text { Sol-A: phosphate buffer } \\
\text { pH } 3.1 \text { and Sol-B: } \\
\text { acetonitrile }\end{array}$ & $\begin{array}{l}\text { Inertsil ODS-3V (150 } \\
\text { mm_4.6 mm,5 } \mu \mathrm{m})\end{array}$ & 225 & 1.5 & $\begin{array}{l}\text { Impurity-B: } \\
0.033\end{array}$ \\
\hline $\begin{array}{l}\text { Present } \\
\text { work }\end{array}$ & HPLC & $\begin{array}{l}\text { Sol. A: } 0.1 \% \mathrm{w} / \mathrm{v} \\
\text { triethylamine, pH } 2.5 \\
\text { Sol. B: ACN: MeOH } \\
(80: 20)(\mathrm{v} / \mathrm{v})\end{array}$ & $\begin{array}{l}\text { Symmetry-C18 ( } 250 \\
\left.\mathrm{~mm} \_4.6 \mathrm{~mm}, 5 \mu \mathrm{m}\right)\end{array}$ & 225 & 1.0 & $\begin{array}{l}\text { LOQ: } \\
\text { PGR-II: } \\
0.00041 \\
\text { PIO-II: } \\
0.000118 \\
\text { N-oxide: } \\
0.000064 \\
\text { Pioglitazone: } \\
0.000109\end{array}$ \\
\hline
\end{tabular}

ACN: Acetonitrile; MeOH: Methanol; LOQ: Limit of quantitation; LOD: Limit of detection

\section{Analytical method validation}

System suitability, system precision, method precision, detection limit, quantitation limit, linearity with regression and range, recovery, specificity/stress study, robustness and solution stability have been accomplished in the method validation study [26].

\section{System suitability}

The system suitability solution was injected and calculated USP resolution for each peak. USP resolution was obtained above 5.0; the results were shown in table 2. (Limit: USP resolution should be more than 5.0 for each peak). The system precision results have been given in table 2 .

Table 2: System suitability results of PGR-II, PIO-II, N-oxide and pioglitazone

\begin{tabular}{llll}
\hline Peak name & RT (min) & RT ratio & USP resolution* \\
\hline PGR-II & 5.51 & 0.35 & - \\
PIO-II & 12.17 & 0.76 & 23.58 \\
Pioglitazone & 15.98 & 1.00 & 10.91 \\
N-oxide & 32.99 & 2.07 & 53.53 \\
\hline
\end{tabular}

RT: Retention time; USP: United states pharmacopoeia; *USP resolution between any peaks should be more than 1.5 . 


\section{System precision}

The precision of an analytical procedure: expresses the nearness of treaty amongst a sequence of quantities obtained from multiple sampling of the same homogeneous sample under the prescribed conditions [26]. The standard solution was repeatedly injected and performed the calculation of \% RSD for each peak. \%RSD was obtained was less than $0.6 \%$ (limit: \%RSD should be less than $4 \%$ ). The system precision results have been given in table 3 .

Table 3: System precision results of PGR-II, PIO-II, N-oxide and pioglitazone

\begin{tabular}{|c|c|c|c|c|}
\hline \multirow[t]{2}{*}{ Injection no. } & \multicolumn{4}{|c|}{ Area observed } \\
\hline & PGR-II & PIO-II & $\mathrm{N}$-oxide & Pioglitazone \\
\hline 1 & 70906 & 40674 & 58335 & 38210 \\
\hline 2 & 71177 & 40876 & 58328 & 38367 \\
\hline 3 & 71172 & 40853 & 58556 & 38487 \\
\hline 4 & 70910 & 40883 & 58194 & 38191 \\
\hline 5 & 70547 & 41004 & 58160 & 38428 \\
\hline 6 & 70587 & 40946 & 58018 & 38168 \\
\hline 7 & 70481 & 40801 & 58157 & 38333 \\
\hline 8 & 70493 & 41295 & 58075 & 38748 \\
\hline 9 & 70536 & 40588 & 57914 & 38697 \\
\hline 10 & 70433 & 41054 & 58010 & 38413 \\
\hline Mean & 70724 & 40897 & 58175 & 38289 \\
\hline SD & 289.8593 & 198.2984 & 190.0509 & 199.1207 \\
\hline$\% \mathrm{RSD}^{*}$ & 0.41 & 0.48 & 0.33 & 0.52 \\
\hline
\end{tabular}

SD: Standard deviation; RSD: Relative standard deviation; * \%RSD limit for 10 injections were should be less than $4 \%$

\section{Method precision}

The precision of an analytical procedure: expresses the nearness of treaty amongst a series of quantities attained from several sampling of the identical homogeneous samples under the prescribed conditions [26]. The method precisions have been performed by six preparations of spiked sample solutions with impurities of PGR-II, PIO-II and N-oxide. The \%RSD for the content of PGR-II, PIO-II and N-oxide was below 6\% (limit: \%RSD for content should be less than 10\%). The \%RSD for the content of impurities was within $6 \%$ in the intermediate precision which was performed by different analysts, column, instrument and day. The table 4 shows the results of method precision data.

\section{Limit of detection and limit of quantitation}

The limit of detection and limit of quantitation was examined based on signal-to-noise ratio method as per the ICH guideline Q2 (R1). The signal to noise ratio for a limit of detection is 3:1 and the limit of quantitation is $10: 1$. This was performed by performing the sequence of dilute solutions with a known concentration limit of detection and limit of quantification has been determined.

The limit of detection for PGR-II, PIO-II, N-oxide and pioglitazone were $0.0007,0.0020,0.0018$, and $0.0011 \%$ respectively. The limit of quantification for PGR-II, PIO-II, N-oxide and pioglitazone were $0.0020,0.0059,0.0054,0.0032 \%$ respectively.

Table 4: Method precision results of PGR-II, PIO-II and N-oxide

\begin{tabular}{llll}
\hline Preparation no. & \% of PGR-II & \% of PIO-II & \% of N-oxide \\
\hline 1 & 0.10 & 0.10 & 0.10 \\
2 & 0.10 & 0.09 & 0.10 \\
3 & 0.10 & 0.09 & 0.10 \\
4 & 0.10 & 0.10 & 0.10 \\
5 & 0.10 & 0.10 & 0.10 \\
6 & 0.10 & 0.10 & 0.10 \\
Mean & 0.10 & 0.10 & 0.10 \\
\%RSD* & 0.0 & 5.2 & 0.0 \\
\hline
\end{tabular}

RSD: Relative standard deviation; *\% RSD for content of impurities was should be less than $10 \%$

Table 5: Linearity data of PGR-II

\begin{tabular}{llll}
\hline Sample No. & \% Level & Concentration $(\boldsymbol{\mu g} / \mathbf{m l})$ & Peak response \\
\hline 1 & LOQ & 0.00041 & 1653 \\
2 & 30 & 0.000616 & 37492 \\
3 & 50 & 0.001026 & 75191 \\
4 & 100 & 0.002052 & 80592 \\
5 & 120 & 0.002462 & 151625 \\
6 & 200 & 0.004104 & 185604 \\
7 & 250 & 0.005130 & 3790 \\
Slope & & 36295198.1479 & -764.8861 \\
Y-intercept & & 0.9985 & 0.9971 \\
Multiple R & &
\end{tabular}

LOQ: limit of quantitation 


\section{Linearity}

The linearity of the analytical procedure: is its capability to attain assessment outcomes which are straightly proportional to the concentration of an analyte in the sample [26]. Linearity was performed from LOQ to $250 \%$ of $2000 \mu \mathrm{g} / \mathrm{ml}$ analyte concentration. The correlation coefficient values have been shown in the table 5-8.
The linearity graphs were shown in fig. 3-6. The values of multiple $R$ and R-square were almost equal to one; this indicates that the developed method was linear. The regression results indicate that the validated method was linear over the total concentration and it was satisfactory for its concentration range from LOQ to $250 \%$. The Rsquare and multiple $\mathrm{R}$ values indicate that the method was linear and it was very close to the origin or close to the ideal theoretical value.

Table 6: Linearity data of PIO-II

\begin{tabular}{llll}
\hline Sample No. & \% level & Concentration $(\boldsymbol{\mu g} / \mathbf{m l})$ & Peak response \\
\hline 1 & LOQ & 0.000118 & 2563 \\
2 & 30 & 0.000600 & 21339 \\
3 & 50 & 0.001000 & 43834 \\
4 & 100 & 0.002000 & 51729 \\
5 & 120 & 0.002400 & 86483 \\
6 & 200 & 0.004000 & 105319 \\
7 & 250 & 0.005000 & \\
Slope & & 21218800.9574 & 501.7382 \\
Y-intercept & & 0.9998 & 0.9995 \\
Multiple R & & & \\
R square & & & \\
\hline
\end{tabular}

LOQ: limit of quantitation

Table 7: Linearity data of $\mathbf{N}$-oxide

\begin{tabular}{llll}
\hline Sample No. & \% level & Concentration $(\boldsymbol{\mu g} / \mathbf{m l})$ & Peak response \\
\hline 1 & LOQ & 0.000064 & 2388 \\
2 & 30 & 0.000597 & 33079 \\
3 & 50 & 0.000996 & 68478 \\
4 & 100 & 0.001992 & 80713 \\
5 & 120 & 0.002390 & 134433 \\
6 & 200 & 0.003983 & 165330 \\
7 & 250 & 0.004979 & \\
Slope & & 33349222.1603 & \\
Y-intercept & & 625.0455 & 0.9999 \\
Multiple R & & 0.9997 & \\
R square & &
\end{tabular}

LOQ: limit of quantitation

Table 8: Linearity data of pioglitazone

\begin{tabular}{llll}
\hline Sample No. & \% Level & Concentration $(\boldsymbol{\mu g} / \mathbf{m l})$ & Peak response \\
\hline 1 & LOQ & 0.000109 & 2362 \\
2 & 30 & 0.000605 & 14950 \\
3 & 50 & 0.001008 & 23884 \\
4 & 100 & 0.002016 & 49743 \\
5 & 120 & 0.002420 & 57282 \\
6 & 200 & 0.004033 & 96295 \\
7 & 250 & 0.005041 & 119350 \\
Slope & & 23707392.2216 & \\
Y-intercept & 393.5717 & \\
Multiple R & & 0.9998 & \\
R square & & 0.9997 & \\
\hline
\end{tabular}

LOQ: limit of quantitation

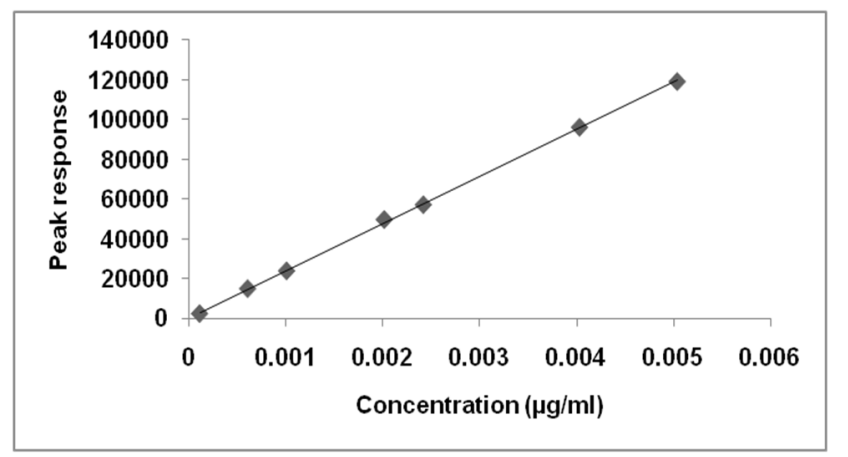

Fig. 3: Linearity graph for PGR-II

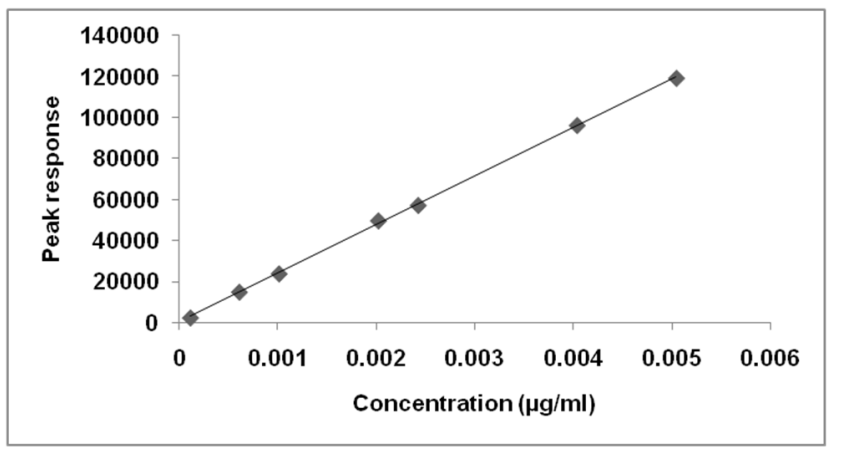

Fig. 4: Linearity graph for PIO-II 


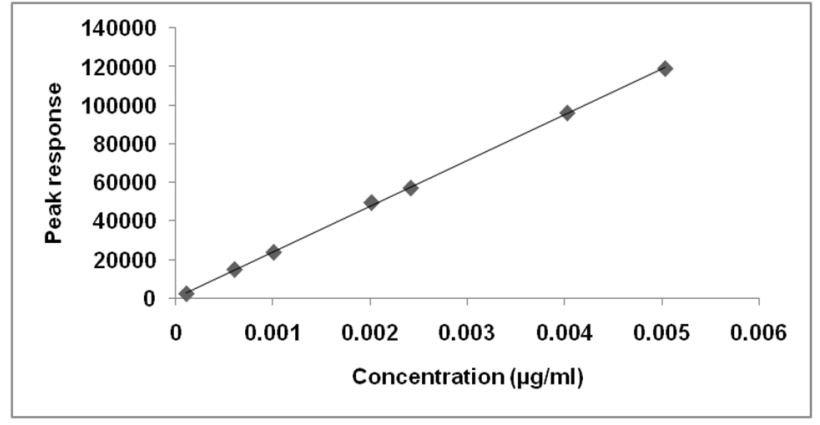

Fig. 5: Linearity graph for N-oxide

\section{Regression}

The regression parameters of PGR-II, PIO-II, N-oxide and pioglitazone have been summarized in table 9 . This explains about the statistical evaluation of ANOVA, confidence intervals and intercepts value with respect to $100 \%$ standard concentration response.

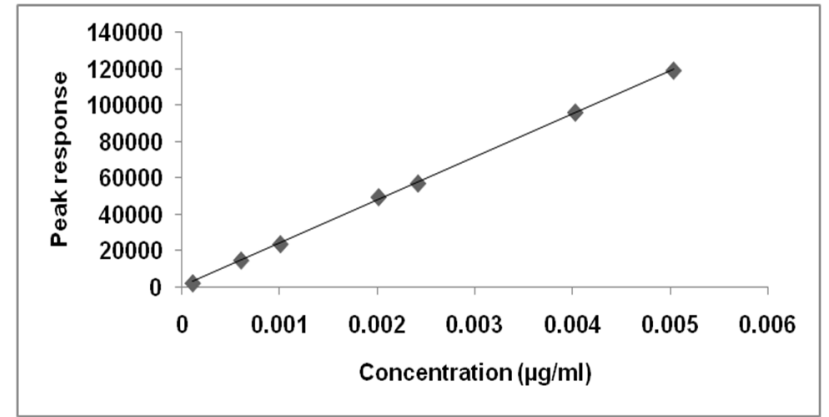

Fig. 6: Linearity graph for pioglitazone

\section{Range}

PGR-II, PIO-II, N-oxide and pioglitazone were established in the range of $2.0-256.5 \%, 5.9-250.0 \%, 3.2-250.2 \%$ and $5.5-252.1 \%$ respectively.

Table 9: Regression statistics

\begin{tabular}{|c|c|c|}
\hline \multirow[t]{2}{*}{ PGR-II } & \multicolumn{2}{|l|}{ ANOVA } \\
\hline & Degree of freedom (df) & Sum of squares (SS) \\
\hline Regression & 1 & 27465435079.1892 \\
\hline Residual & 5 & 80876504.2393 \\
\hline Total & 6 & 27546311583.4286 \\
\hline \multirow[t]{2}{*}{ PGR-II } & Confidence Intervals & \\
\hline & Lower 95\% & Upper $95 \%$ \\
\hline Intercept & -7103.8007 & 5574.0285 \\
\hline X Variable 1 & 34031004.6031 & 38559391.6926 \\
\hline \multirow[t]{2}{*}{ PIO-II } & ANOVA & \\
\hline & Degree of freedom (df) & Sum of squares (SS) \\
\hline Regression & 1 & 8772141115.7928 \\
\hline Residual & 5 & 4127381.6358 \\
\hline Total & 6 & 8776268497.4286 \\
\hline \multirow[t]{2}{*}{ PIO-II } & Confidence Intervals & \\
\hline & Lower 95\% & Upper $95 \%$ \\
\hline Intercept & -942.2478 & 1945.7241 \\
\hline X Variable 1 & 20689683.8784 & 21747918.0363 \\
\hline \multirow[t]{2}{*}{ N-oxide } & ANOVA & \\
\hline & Degree of freedom (df) & Sum of squares (SS) \\
\hline Regression & 1 & 21732116399.7025 \\
\hline Residual & 5 & 5734902.0117 \\
\hline Total & 6 & 21737851301.7143 \\
\hline \multirow[t]{2}{*}{$\mathrm{N}$-oxide } & Confidence Intervals & \\
\hline & Lower 95\% & Upper $95 \%$ \\
\hline Intercept & -1067.2941 & 2317.3851 \\
\hline X Variable 1 & 32726428.5752 & 33972015.7453 \\
\hline \multirow[t]{2}{*}{ Pioglitazone } & ANOVA & \\
\hline & Degree of freedom (df) & Sum of squares (SS) \\
\hline Regression & 1 & 11154571681.1543 \\
\hline Residual & 5 & 3631851.7028 \\
\hline Total & 6 & 11158203532.8571 \\
\hline \multirow{2}{*}{ Pioglitazone } & Confidence Intervals & \\
\hline & Lower 95\% & Upper 95\% \\
\hline Intercept & -959.5021 & 1746.6455 \\
\hline X Variable 1 & 23215615.6658 & 24199168.7775 \\
\hline
\end{tabular}

ANOVA: Analysis of variance; Regression data shows that the validated method was statistically proven.

\section{Accuracy}

The accuracy of an analytical procedure: expresses the nearness of treaty amongst the value which is accepted either as a conventional factual value or a recognized reference value and the significance found. For the quantitative approaches, at least nine determinations across the specified range should be obtained [26]. The accuracy percentage for PGR-II, PIO-II and N-oxide were $98-105 \%$ for drug substance from LOQ to $250.0 \%$ level. This result indicates that the method was accurate and appropriate as the mean accuracy value was within the limit (80-120\%).

\section{Specificity}

Specificity is the capability to judge the compound of interest unequivocally in the presence of components, which may be anticipated to be existent. Typically, these might include impurities, degradants, matrix, etc. [26]. Specificity of the method was 
demonstrated by the peak purity, (i.e. the purity angle is lesser than the purity threshold) by way of the diode array detector for degraded samples. The specificity of the method was established with the pioglitazone in the existence of related impurities namely PGR-II, PIO-II and N-oxide. To prove the specificity; all forced degraded studied samples were performed at a test concentration of $2000 \mu \mathrm{g} / \mathrm{ml}$. The peak purity analysis was homogenous for PGR-II, PIO-II and N-oxide. There was no interference observed from blank peaks and impurities. There was no secondary peak aroused from degraded samples. The results of forced degradation study indicate that the method was stability indicating. The impurity PGR-II and PIO-II were process related impurities and $\mathrm{N}$-oxide was degradation impurity.

\section{Robustness}

By careful variation in chromatographic conditions, the resolution between PGR-II, PIO-II, N-oxide and pioglitazone were evaluated. The mobile phase flow rate was $1.0 \mathrm{ml} / \mathrm{min}$. To check the effect of flow rate on the resolution, 0.1 units changed it from 0.9 to 1.1 $\mathrm{ml} / \mathrm{min}$. The column oven temperature was $45^{\circ} \mathrm{C}$. To check the effect of temperature on the resolution, 5 units changed it from 40 ${ }^{\circ} \mathrm{C}$ to $50{ }^{\circ} \mathrm{C}$. The $\%$ of mobile phase-A composition was $80 \%$. To check the effect of mobile phase composition on the resolution, $2 \%$ units changed it from $78 \%$ to $82 \%$. The $\%$ of mobile phase-B composition was $20 \%$. To check the effect of mobile phase composition on the resolution, $2 \%$ units changed it from $18 \%$ to $22 \%$. The resolution between impurities and pioglitazone was greater than 5 in all the varied chromatographic conditions carried out (flow rate, the addition of trifluoroacetic acid and column temperature). The result shows that the method was considered robust.

\section{Solution stability}

The solution stability of pioglitazone and its impurities was carried out by freshly prepared standard and sample solution in a tightly closed volumetric flask at the room temperature $\left(22-27^{\circ} \mathrm{C}\right)$ as well as in the refrigerator at $2-8^{\circ} \mathrm{C}$ for initial, 24 and $48 \mathrm{~h}$. The result of solution stability shows that the solution was stable up to $48 \mathrm{~h}$ at room temperature $\left(22-27{ }^{\circ} \mathrm{C}\right)$ and also at $2-8{ }^{\circ} \mathrm{C}$ in the refrigerator.

\section{DISCUSSION}

The USP resolution between PIO-II and pioglitazone was 15.9 which were 5 times more than that the obtained value of the research article [27]. The system precision results were very well within the acceptance criteria, i.e., \%RSD for PIO-II, PGR-II, $\mathrm{N}$-oxide and pioglitazone were observed within $0.5 \%$. The \%RSD value was obtained by the research work [27] was above $0.5 \%$ when compared to the present research study which was observed less than $0.5 \%$. Moreover, the \%RSD obtained in the validation was very less when compared to the previously published articles. The limit of detection and limit of quantitation results indicated that the method was sensitive to determine the content PGR-II, PIO-II and N-oxide in the sample of pioglitazone drug substance.

The method precision results show that the developed method was very precise with the addition of impurities in the presence of pioglitazone hydrochloride when compared to the previously established works. The detection and quantitation limits of PIO-II, PGR-II, N-oxide and pioglitazone were observed low when compared to the previously published article [27] though the study was performed for several impurities. The developed method was linear from very low level to high level when compared to the previously published article [27]. The accuracy of the method has been demonstrated by the presence of impurities in pioglitazone hydrochloride at the specified levels with respect to the test concentration; the results show that the method was very accurate at the LOQ level itself. The specificity, solution stability and robustness of the method show that the impurity PGR-II and PIO-II were process related impurities and N-oxide was degradation impurity. This indicates that the equipment was suitable, accurate, precise, sensitive and fit for study.

\section{CONCLUSION}

The developed RP-HPLC method was developed and validated as per ICH guidelines in terms of system suitability, system precision, method precision, specificity/stress studies, accuracy, linearity, robustness, solution stability, limit of detection and limit of quantitation for the quantitative estimation of related substances of pioglitazone hydrochloride drug substance. The correlation coefficients were greater than 0.99 . The precision results were good enough to say that the method developed is precise and reproducible. Accuracy studies revealed that mean recoveries after spiking experiments were between 98 and 105\%, indicative of accurate method. Degradation studies reveal that the developed method was stability indicating hence, this method can easily and conveniently adopt for routine quality control analysis of the determination of related substances of pioglitazone drug substances in quality control laboratories.

\section{FUNDING}

The study did not receive any funding.

\section{ACKNOWLEDGEMENT}

The authors acknowledge the support provided by the research scholars of the chemistry department, St. Peter's University.

\section{CONFLICTS OF INTERESTS}

There is no conflict of interest to declare

\section{REFERENCES}

1. Belfort R, Harrison SA, Brown K, Darland C, Finch J, Hardies J, et al. A placebo-controlled trial of pioglitazone in subjects with nonalcoholic steatohepatitis. N Engl J Med 2006;355:2297-307.

2. http://www.accessdata.fda.gov/drugsatfda_docs/label/2011/ 021073s043s044lbl.pdf. [Last accessed on 20 Nov 2016]

3. Radhakrishna T, Rao DS, Reddy GO. Determination of pioglitazone hydrochloride in bulk and pharmaceutical formulations by HPLC and MEKC methods. J Pharm Biomed Anal 2002;29:593-607.

4. Reddy GRK, Rao VSN. Development and validation of stability indicating assay method for pioglitazone drug substance by reverse phase HPLC. J Global Trends Pharm Sci 2012;3:584-96.

5. Sharma S, Sharma MC, Chaturvedi SC. Study of stressed degradation behaviour of pioglitazone hydrochloride in bulk and pharmaceutical formulation by HPLC assay method. J Optoelectron Biomed Mater 2010;1:17-24.

6. Saber AMRL. Determination of pioglitazone hydrochloride in tablets by high-performance liquid chromatography. Pak J Anal Environ Chem 2008;9:118-21.

7. Jedlicka A, Klimes J, Grafnetterova T. Reversed-phase HPLC methods for purity test and assay of pioglitazone hydrochloride in tablets. Pharmazie 2004;59:178-82.

8. Wanjari DB, Gaikwad NJ. Stability indicating a RP-HPLC method for determination of pioglitazone from tablets. Indian J Pharm Sci 2005;67:256-8.

9. Sane RT, Menon SN, Inamdar S, Mote M, Gundi G. Simultaneous determination of pioglitazone and glimepiride by highperformance liquid chromatography. Chromatographia 2004;59:451-3.

10. Swapna J, Madhu C, Srivani S, Sumalatha M, Nehalatha Y, Anusha Y. Analytical method development and method validation for the simultaneous estimation of metformin hydrochloride and pioglitazone hydrochloride in tablet dosage form by RP-HPLC. Asian J Pharm Anal 2012;2:85-9.

11. Karthik A, Subramanian G, Rao CM, Bhat K, Ranjithkumar A, Musmade $\mathrm{P}$, et al. Simultaneous determination of pioglitazone and glimepiride in bulk drug and pharmaceutical dosage form by RP-HPLC method. Pak J Pharm Sci 2008;21:421-5.

12. Jain D, Jain S, Maulik A. Simultaneous estimation of metformin hydrochloride, pioglitazone hydrochloride and glimepiride by RP-HPLC in tablet formulation. J Chromatogr Sci 2008;46:501-4.

13. Sarat M, Krishna PM, Rambabu C. RP-HPLC method for estimation of saxagliptin and pioglitazone in tablets. Int Res J Pharm 2012;3:399-402. 
14. Kalyankar TM, Badgujar MR, Kakde RB. Simultaneous determination of pioglitazone $\mathrm{HCl}$ and glimepiride by RP-HPLC in pharmaceutical dosage form. J Pharm Res 2010;3:3078-80.

15. Havaldar FH, Vairal DL. Simultaneous estimation of glimepiride, rosiglitazone and pioglitazone hydrochloride in the pharmaceutical dosage form. J Chem 2010;7:1326-33.

16. Shankar MB, Modi VD, Shah DA, Bhatt KK, Mehta RS, Patel BJ. Estimation of pioglitazone hydrochloride and metformin hydrochloride in tablets by derivative spectrophotometry and liquid chromatographic methods. J AOAC Int 2005;88:1167-72.

17. Nirupa G, Tirupathi UM. RP-HPLC analytical method development and validation for simultaneous estimation of three drugs: glimepiride, pioglitazone and metformin and its pharmaceutical dosage forms. J Chem 2013;2013:1-8.

18. Sakuntala MSV, Prasad SVUM, Devi SS, Yadav K, Reddy KS. A RP-HPLC method development and validation for the simultaneous estimation of glimepiride and pioglitazone $\mathrm{HCl}$ in tablet dosage forms. J Chem Pharm Res 2012;4:154-9.

19. Navaneethan G, Karunakaran K, Elango KP. Simultaneous estimation of pioglitazone, glimepiride and glimepiride impurities in combination drug product by a validated stabilityindicating RP-HPLC method. J Chil Chem Soc 2011;56:815-8.

20. Shweta H, Dhaneshwar S. Development and validation of a HPLC method for the determination of metformin $\mathrm{HCl}$, gliclazide and pioglitazone hydrochloride in the multi-component formulation. Webmed Central Pharm Sci 2010;10:1-16.

21. Vinodkumar K, Sudhakar M, Padmanabhareddy Y, Swapna A, Rajanisekhar V. Method development and validation for simultaneous estimation of pioglitazone and glimepiride in tablet dosage form by RP-HPLC and UV-spectrophotometric method. J Curr Pharma Res 2011;2:404-10.

22. Premanand DC, Senthilkumar KL, Senthilkumar B, Saravanakumar M, Thirumurthy R. A new RP-HPLC method development and validation for simultaneous estimation of telmisartan and pioglitazone in pharmaceutical dosage form. Int J Chem Tech Res 2011;3:448-54.

23. Lakshmi KS, Rajesh T, Sharma S, Lakshmi S. Development and validation of liquid chromatographic and UV derivative spectrophotometric methods for the determination of metformin, pioglitazone and glimepiride in pharmaceutical formulations. Pharma Chem 2009;1:238-46.

24. Sharma MC, Sharma S, Kohli DV, Chaturvedi SC. Micellar liquid chromatographic analytical method development and validation of determination of atorvastatin calcium and pioglitazone in tablet dosage form. Pharma Chem 2010;2:273-80.

25. Madhukar A, Naresh K, Naveenkumar CH, Sandhya N, Prasanna P. Rapid and sensitive RP-HPLC analytical method development and validation of pioglitazone hydrochloride. Pharm Lett 2011;3:128-32.

26. http://www.ich.org/fileadmin/Public_Web_Site/ICH_Products /Guidelines/Quality/Q2_R1/Step4/Q2_R1_Guideline.pdf. [Last accessed on 20 Nov 2016]

27. Rashmitha N, Hiriyanna SG, Sreenivasarao $\mathrm{CH}$, Chandrasekhar reddy $\mathrm{K}$, Harikiran M, Hemantkumarsharma, et al. A validated stability indicating HPLC method for the determination of impurities in pioglitazone hydrochloride. Pharma Chem 2010;2:426-33.

28. Balaji N, Sayeeda S. Ultra-high performance liquid chromatographic determination of genotoxic impurities in febuxostat drug substance and products. Asian J Pharm Clin Res 2017;10:324-30.

29. Hisham E, Omnia AI, Wafaa SH, Abdalla S. Development and validation of a stability-indicating HPLC-UV method for the determination of pioglitazone hydrochloride and metformin hydrochloride in bulk drug and combined dosage form. Asian J Pharm Clin Res 2013;6:116-20.

30. Balaji N, Sayeeda S. Trace level determination and quantification of potential genotoxic impurities in dasatinib drug substance by UHPLC/infinity LC. Int J Pharm Pharm Sci 2016;8:209-16.

31. Mallikarjuna RN, Gowrisankar D. RP-HPLC method for simultaneous estimation and stability indicating the study of metformin and linagliptin in pure and pharmaceutical dosage forms. Int J Pharm Pharm Sci 2015;7:191-7.

\section{How to cite this article}

- N Balaji, Sayeeda Sultana. Sensitive determination of related substances in pioglitazone hydrochloride by HPLC. Int J Appl Pharm 2017;9(2):34-41. 